\title{
A NOTE ON THE SIMULATION OF THE GINIBRE POINT PROCESS
}

\author{
LAURENT DECREUSEFOND, ${ }^{*}$ \\ IAN FLINT *** AND \\ ANAIS VERGNE, ${ }^{*}$ Télécom Paristech
}

\begin{abstract}
The Ginibre point process (GPP) is one of the main examples of determinantal point processes on the complex plane. It is a recurring distribution of random matrix theory as well as a useful model in applied mathematics. In this paper we briefly overview the usual methods for the simulation of the GPP. Then we introduce a modified version of the GPP which constitutes a determinantal point process more suited for certain applications, and we detail its simulation. This modified GPP has the property of having a fixed number of points and having its support on a compact subset of the plane. See Decreusefond et al. (2013) for an extended version of this paper.
\end{abstract}

Keywords: Determinantal point process; Ginibre point process; point process simulation

2010 Mathematics Subject Classification: Primary 60G55; 60K35; 60G60

Secondary $15 \mathrm{~A} 52$

\section{Introduction}

Determinantal point processes (DPP) form a class of point processes which exhibit repulsion and are used to model a wide variety of phenomena. After their introduction by Macchi [11], they have been studied in depth from a probabilistic point of view; see [15] and [16] for an overview of their mathematical properties. Other than modeling fermion particles (see the account of the determinantal structure of fermions in [17] and also [16] for other examples), they are known to appear in many branches of stochastic matrix theory (see, e.g. [16] or the thorough overview of [1]) and in the study of the 0s of Gaussian analytic functions; see [7]. The Ginibre point process (GPP) in particular was first introduced in [4] and arises in many problems revolving around DPPs. To be more specific, the eigenvalues of a Hermitian matrix with complex Gaussian entries (which is a subclass of the so-called Gaussian unitary ensemble (GUE)) are known to form a GPP (or rather, a so-called truncated GPP). The GPP models the positions of charges of a two-dimensional Coulomb gas in a harmonic oscillator potential, at a temperature corresponding to $\beta=2$; see [4]. Furthermore, the GPP is the natural extension of the Dyson point process to the complex plane. The Dyson model is a DPP on $\mathbb{R}$ which is of central importance, as it appears as the bulk-scaling limit of a large class of DPPs; see [2].

The simulation of a general DPP is mostly unexplored, and was in fact initiated in [6] wherein the authors presented a practical algorithm for the simulation of a DPP. Theoretical discussion of the aforementioned algorithm as well as statistical aspects have also been explored in [8]. More specifically, the GPP as a model has spiked interest since its introduction in [4]. The simulation procedure which is hinted in [4] was fully developed in [10]. To the best of the

Received 28 November 2013; revision received 12 September 2014.

* Postal address: Télécom ParisTech, 46, rue Barrault, 75634 Paris cedex 13, France.

** Email address: iflint@ntu.edu.sg 
authors' knowledge, the first use of the GPP as a model traces back to [9]. More recently, in [12], [18], and [19], different authors have used the GPP to model phenomena arising in networking. Indeed, this particular model has many advantages with regards to applications. It is invariant with respect to rotations and translations, which gives us a natural compact subset on which to simulate it; namely, the ball centered at the origin. Moreover, the electrostatic repulsion between particles is found to be fitting for many applications. The main problem that arises in the usual simulation procedure of the GPP is that although the eigenvalues of matrices in the GUE ensemble form a (so-called truncated) GPP, these eigenvalues are not compactly supported, although after renormalization, they tend to be compactly supported as $N$, the size of the GUE matrix, tends to $\infty$ (this is known as the circular law in stochastic matrix theory). Additionally, we remark that the usual simulation techniques of the GPP do not transpose well to the Ginibre $\alpha$-DPP, defined in [15]. Indeed, in order to simulate the associated $\alpha$-DPP, the general algorithms of [6] are required for the simulation. We thus introduce a modified version of the GPP, which tends weakly to the usual GPP as the parameter $N$ tends to $\infty$. Moreover, since our point process is a projection DPP, it is defined on a compact subset and its simulation follows from a simplified version of the algorithm in [6]. As a pleasant side-effect, the simulation procedure may also be extended to the $\alpha$-DPP case.

We proceed as follows. We start in Section 2 by general definitions relating to point processes. In Section 3 we present more specifically the GPP, and discuss its usual simulation. We then introduce our modified version of the GPP, gives its Janossy density, prove its convergence to the GPP, and conclude by discussing its simulation.

\section{Preliminary notions of point process theory}

Let $E$ be a Polish space, and $\mathscr{B}$ the Borel $\sigma$-algebra on $E$. Let $\lambda$ be a Radon measure on $(E, \mathscr{B})$. For a set $A \subset E$, define $|A|$ to be the cardinal of $A$, with the convention $|A|=+\infty$ if the set $A$ is not finite. Let $\mathcal{X}$ be the space of locally finite subsets of $E$, sometimes called the configuration space:

$$
X=\{\xi \subset E:|\Lambda \cap \xi|<\infty \text { for any compact set } \Lambda \subset E\} .
$$

Elements of $X$ are called configurations in what follows. The configuration space $X$ is naturally topologized by the vague topology, which is the weakest topology such that for all continuous and compactly supported functions $f$ on $E$, the mapping $\xi \mapsto\langle f, \xi\rangle:=\sum_{y \in \xi} f(y)$ is continuous. We denote by $\mathcal{F}$ the corresponding Borel $\sigma$-algebra. Next, let $x_{\Lambda}=\{\xi \subset$ $\Lambda:|\xi|<\infty\}$ be the space of all finite configurations on a compact set $\Lambda$ of $E$, equipped with the trace $\sigma$-algebra $\mathcal{F}_{\Lambda}=\mathcal{F} \mid x_{\Lambda}$. A point process is defined as a probability measure $\mu$ on $(\mathcal{X}, \mathcal{F})$. A point process $\mu$ is said to have correlation functions $\left(\rho_{n}\right)_{n \in \mathbb{N}}$ if for any $A_{1}, \ldots, A_{n}$ disjoint bounded Borel subsets of $E$,

$$
\int_{X} \prod_{i=1}^{n} \xi\left(A_{i}\right) \mu(\mathrm{d} \xi)=\int_{A_{1} \times \cdots \times A_{n}} \rho_{n}\left(x_{1}, \ldots, x_{n}\right) \lambda\left(\mathrm{d} x_{1}\right) \cdots \lambda\left(\mathrm{d} x_{n}\right) .
$$

Recall that $\rho_{1} \mathrm{~d} \lambda$ is the particle density and $\rho_{n}\left(x_{1}, \ldots, x_{n}\right) \lambda\left(\mathrm{d} x_{1}\right) \cdots \lambda\left(\mathrm{d} x_{n}\right)$ is the probability of finding a particle in the vicinity of each $x_{i}, i=1, \ldots, n$. For any compact set $\Lambda \subset E$, we also define the Janossy density $j_{\Lambda}$ of $\mu$, which is such that for any measurable integrable $f: \chi_{\Lambda} \rightarrow \mathbb{R}$

$$
\int_{X} f(\xi) \mu(\mathrm{d} \xi)=\sum_{n \geq 0} \frac{1}{n !} \int_{\Lambda^{n}} f\left(\left\{x_{1}, \ldots, x_{n}\right\}\right) j_{\Lambda}\left(\left\{x_{1}, \ldots, x_{n}\right\}\right) \lambda\left(\mathrm{d} x_{1}\right) \cdots \lambda\left(\mathrm{d} x_{n}\right) .
$$


In what follows, we write $j_{\Lambda}^{n}\left(x_{1}, \ldots, x_{n}\right):=j_{\Lambda}\left(\left\{x_{1}, \ldots, x_{n}\right\}\right)$ for the $n$th Janossy density, i.e. the associated symmetric function of $n$ variables, for a configuration of size $n \in \mathbb{N}$. In fact, $j_{\Lambda}^{n}\left(x_{1}, \ldots, x_{n}\right)=n ! p_{n} f_{\Lambda}^{n}\left(x_{1}, \ldots, x_{n}\right)$, where $p_{n}=\mu(|\xi \cap \Lambda|=n)$ and $f_{\Lambda}^{n}$ is the joint density of the points, given that the number of points is $n$.

\section{Simulation of the GPP}

\subsection{Overview of the standard methods}

For details on DPPs, we refer the reader to [15] and [16]. The GPP is defined as the determinantal process on $\mathbb{C}$ with kernel

$$
K(x, y)=\frac{1}{\pi} \mathrm{e}^{x \bar{y}} \mathrm{e}^{-\left(|x|^{2}+|y|^{2}\right) / 2}, \quad x, y \in \mathbb{C},
$$

with respect to $\mathrm{d} \lambda:=\mathrm{d} \ell$, the Lebesgue measure on $\mathbb{C}$ (i.e. $\mathrm{d} \ell(x)=\mathrm{d} a \mathrm{~d} b$, when $x=a+\mathrm{i} b$ ). In (1), $\bar{z}$ denotes the conjugate of a complex $z \in \mathbb{C}$. The kernel (1) is decomposed in the basis of its eigenvectors as

$$
K(x, y)=\sum_{n \geq 0} \phi_{n}(x) \overline{\phi_{n}(y)}, \quad x, y \in \mathbb{C},
$$

where $\phi_{n}(x):=(1 / \sqrt{\pi n !}) \mathrm{e}^{-|x|^{2} / 2} x^{n}$, for $n \in \mathbb{N}$ and $x \in \mathbb{C}$. It can be easily checked that $\left(\phi_{n}\right)_{n \in \mathbb{N}}$ is an orthonormal family of $L^{2}(\mathbb{C}, \mathrm{d} \ell)$. In fact, $\left(\phi_{n}\right)_{n \in \mathbb{N}}$ is a dense subset of $L^{2}(\mathbb{C}, \mathrm{d} \ell)$. The GPP is known to be stationary and isotropic, which as mentioned already makes it useful for modeling.

First, we mention the truncated Ginibre point process (TGPP), defined for $N \geq 0$ by its kernel

$$
K^{N}(x, y)=\sum_{n=0}^{N-1} \phi_{n}(x) \overline{\phi_{n}(y)}, \quad x, y \in \mathbb{C},
$$

which is in fact a truncation of the sum in (2). As it is a projection kernel (see, e.g. [16]), the TGPP has $N$ points almost surely. It is clearly not translation invariant anymore; however it remains isotropic. From a physical point of view, the TGPP is the distribution of $N$ polarized electrons in a perpendicular magnetic field, filling the $N$ lowest Landau levels, as is remarked in [13].

The simulation of the TGPP with kernel given by (3) is in fact quite well known. Since it has $N$ points almost surely, one only needs to simulate the positions of the $N$ points. It was proven in [4] that the eigenvalues of an $N \times N$ Hermitian matrix with complex Gaussian entries are distributed according to a TGPP. Generating such a Hermitian matrix and computing its eigenvalues numerically is by far the most efficient method to simulate the TGPP.

Recall that the joint law of the TGPP is computed in [7, Theorem 4.3.10] as

$$
p\left(x_{1}, \ldots, x_{N}\right)=\frac{1}{\pi^{N}} \prod_{p=0}^{N} \frac{1}{p !} \mathrm{e}^{-\sum_{p=1}^{N}\left|x_{p}\right|^{2}} \prod_{1 \leq p<q \leq N}\left|x_{p}-x_{q}\right|^{2}, \quad x_{1}, \ldots, x_{n} \in \mathbb{C},
$$

which has support on $\mathbb{C}^{N}$. Thus, one runs into a practical problem when simulating this point process: the support of its law is the whole of $\mathbb{C}^{N}$. Moreover, projecting onto a compact subset randomizes the number of points in the point process. Thus, this first point process is mostly useful in applications where the point process has a fixed number of points that need not be in a fixed compact subset of $\mathbb{C}$. 


\subsection{Truncated Ginibre process on a compact subset}

In this section we begin by studying the truncated Ginibre point process on a compact subset (TGPPC), and specifically discuss the most judicious choice of the compact subset onto which to project. Namely, define $K_{R}^{N}$ to be the projection of the integral operator associated to the kernel $K^{N}$ (defined in (3)) onto $L^{2}\left(\mathscr{B}_{R}, \mathrm{~d} \ell\right.$ ), where $\mathscr{B}_{R} \subset \mathbb{C}$ is the closed ball centered at the origin and of radius $R$. The associated kernel may be explicitly computed (see [14]) as

$$
K_{R}^{N}\left(x_{1}, x_{2}\right)=\sum_{n=0}^{N-1} \lambda_{n}^{R} \phi_{n}^{R}\left(x_{1}\right) \overline{\phi_{n}^{R}\left(x_{2}\right)}, \quad x_{1}, x_{2} \in \mathcal{B}_{R},
$$

where $\phi_{n}^{R}(x):=Z_{R, n}^{-1} \phi_{n}(x) \mathbf{1}_{\left\{x \in \mathcal{B}_{R}\right\}}, n \in \mathbb{N}, x \in \mathbb{C}$, and

$$
\lambda_{n}^{R}:=\int_{\mathcal{B}_{R}}\left|\phi_{n}(x)\right|^{2} \mathrm{~d} \ell(x)=Z_{R, n}^{2}=\frac{\gamma\left(n+1, R^{2}\right)}{n !} .
$$

Here, $\gamma$ is the lower incomplete gamma function defined as $\gamma(z, a):=\int_{0}^{a} \mathrm{e}^{-t} t^{z-1} \mathrm{~d} t$ for $z \in \mathbb{C}$ and $a \geq 0$. We emphasize that the decomposition (4) does not hold in general for the projection on a compact set, but is rather due to the fact that $\left(\phi_{n}^{R}(\cdot)\right)_{n \geq 0}$ is still an orthonormal family of $L^{2}\left(B_{R}, \mathrm{~d} \ell\right)$.

Since the sum in (4) has $N$ terms, the TGPPC has less than $N$ points almost surely (see, e.g. [16]). Therefore, it suffices to calculate the Janossy densities $j_{R}^{0}, \ldots, j_{R}^{N}$ to characterize the distribution of the TGPPC. These are given by the next proposition.

Proposition 1. The DPP with kernel given by (4) has less than $N$ points almost surely, and its Janossy densities are given by

$$
\begin{aligned}
j_{R}^{k}\left(x_{1}, \ldots, x_{k}\right)= & \frac{1}{\pi^{k}}\left(\prod_{p=0}^{N-1} \frac{\Gamma\left(p+1, R^{2}\right)}{p !}\right) \mathrm{e}^{-\sum_{p=1}^{k}\left|x_{p}\right|^{2}} \prod_{1 \leq i<j \leq k}\left|x_{i}-x_{j}\right|^{2} \\
& \times \sum_{\left\{i_{1}, \ldots, i_{k}\right\} \subset\{1, \ldots, N\}}\left(\prod_{p=1}^{k} \frac{1}{\Gamma\left(i_{p}, R^{2}\right)}\right)\left|s_{\lambda\left(i_{1}, \ldots, i_{k}\right)}\left(x_{1}, \ldots, x_{k}\right)\right|^{2}
\end{aligned}
$$

for $0 \leq k \leq N, \lambda\left(i_{1}, \ldots, i_{k}\right):=\left(i_{k}-k, \ldots, i_{2}-2, i_{1}-1\right)$, and $x_{1}, \ldots, x_{k} \in B_{R}$. Here, $s$ is the Schur polynomial defined as

$$
s_{\left(i_{1}, \ldots, i_{k}\right)}\left(x_{1}, \ldots, x_{k}\right):=\frac{V_{i_{k}+1, i_{k-1}+2, \ldots, i_{1}+k}\left(x_{1}, \ldots, x_{k}\right)}{V_{1, \ldots, k}\left(x_{1}, \ldots, x_{k}\right)}
$$

where, for any $i_{1}, \ldots, i_{k} \in \mathbb{N}$,

$$
V_{i_{1}, \ldots, i_{k}}\left(x_{1}, \ldots, x_{k}\right):=\operatorname{det}\left(\left(x_{h}^{i_{p}-1}\right)_{1 \leq p, h \leq k}\right)
$$

is known in the literature as the generalized Vandermonde determinant, while noting that $V_{1, \ldots, k}\left(x_{1}, \ldots, x_{k}\right)$ is the usual Vandermonde determinant. The Schur polynomial is known to be symmetric, and is a sum of monomials; see, e.g. [5] for further details.

Proof. Since the spectrum of $K_{R}^{N}$ is included in $(0,1)$, the local interaction operator $J^{N}\left[\mathcal{B}_{R}\right]$ (defined by $\left(I-K_{R}^{N}\right)^{-1} K_{R}^{N}$ ) associated to the TGPPC can be decomposed in the same basis 
of eigenvectors as $K_{R}^{N}$ by

$$
J^{N}\left[\mathscr{B}_{R}\right]\left(x_{1}, x_{2}\right)=\sum_{n=0}^{N-1} \frac{\gamma\left(n+1, R^{2}\right)}{\Gamma\left(n+1, R^{2}\right)} \phi_{n}^{R}\left(x_{1}\right) \overline{\phi_{n}^{R}\left(x_{2}\right)}, \quad x_{1}, x_{2} \in \mathscr{B}_{R},
$$

where $\Gamma$ is the upper incomplete gamma function defined as $\Gamma(z, a):=\int_{a}^{\infty} \mathrm{e}^{-t} t^{z-1} \mathrm{~d} t$ for $z \in \mathbb{C}$ and $a \geq 0$, which by definition verifies $\gamma(\cdot, a)+\Gamma(\cdot, a)=\Gamma(\cdot)$ for all $a \geq 0(\Gamma(\cdot)$ is the usual gamma function). Here, we note that $j_{R}^{N}$ can be computed easily since the determinant which appears is a Vandermonde determinant:

$$
\operatorname{det}\left(J^{N}\left[\mathscr{B}_{R}\right]\left(x_{i}, x_{j}\right)\right)_{1 \leq i, j \leq N}=\frac{1}{\pi^{N}} \prod_{p=0}^{N-1} \frac{1}{\Gamma\left(p+1, R^{2}\right)} \mathrm{e}^{-\sum_{p=1}^{N}\left|x_{p}\right|^{2}} \prod_{1 \leq p<q \leq N}\left|x_{p}-x_{q}\right|^{2}
$$

for $x_{1}, \ldots, x_{N} \in \mathscr{B}_{R}$. Moreover, the hole probability, i.e. the probability of having no point in $\mathscr{B}_{R}$, can be expressed as

$$
\operatorname{det}\left(I-K_{R}^{N}\right)=\prod_{n=0}^{N-1}\left(1-\lambda_{n}^{R}\right)=\prod_{n=0}^{N-1} \frac{\Gamma\left(n+1, R^{2}\right)}{n !} .
$$

By [15, Lemma 3.4], the $N$ th Janossy density has the following form:

$$
\begin{aligned}
j_{R}^{N}\left(x_{1}, \ldots, x_{N}\right) & =\operatorname{det}\left(I-K_{R}^{N}\right) \operatorname{det}\left(J^{N}\left[\mathscr{B}_{R}\right]\left(x_{i}, x_{j}\right)\right)_{1 \leq i, j \leq N} \\
& =\frac{1}{\pi^{N}} \prod_{p=0}^{N-1} \frac{1}{p !} \mathrm{e}^{-\sum_{p=1}^{N}\left|x_{p}\right|^{2}} \prod_{1 \leq p<q \leq N}\left|x_{p}-x_{q}\right|^{2} \quad \text { for } x_{1}, \ldots, x_{N} \in \mathscr{B}_{R} .
\end{aligned}
$$

Now, if we take $k<N$, we have again

$$
J^{N}\left[\mathscr{B}_{R}\right]\left(x_{1}, \ldots, x_{k}\right)=A^{N}\left(x_{1}, \ldots, x_{k}\right) A^{N}\left(x_{1}, \ldots, x_{k}\right)^{*},
$$

where this time $A^{N}\left(x_{1}, \ldots, x_{k}\right)$ is a rectangular $k \times N$ matrix defined by

$$
A_{p h}^{N}\left(x_{1}, \ldots, x_{k}\right):=\sqrt{\frac{\gamma\left(h, R^{2}\right)}{\Gamma\left(h, R^{2}\right)}} \phi_{h-1}^{R}\left(x_{p}\right) \text { for } 1 \leq p \leq k, 1 \leq h \leq N .
$$

Hence, by application of the Cauchy-Binet formula, we have

$$
\operatorname{det} J\left[\mathscr{B}_{R}\right]\left(x_{1}, \ldots, x_{k}\right)=\sum_{\left\{i_{1}, \ldots, i_{k}\right\} \subset\{1, \ldots, N\}}\left|\operatorname{det} A^{i_{1}, \ldots, i_{k}}\left(x_{1}, \ldots, x_{k}\right)\right|^{2},
$$

where we have set, for $1 \leq p, h \leq k$,

$$
A_{p h}^{i_{1}, \ldots, i_{k}}\left(x_{1}, \ldots, x_{k}\right):=\sqrt{\frac{\gamma\left(i_{h}, R^{2}\right)}{\Gamma\left(i_{h}, R^{2}\right)}} \phi_{i_{h}-1}^{R}\left(z_{p}\right),
$$

which is a square matrix. We now consider a fixed $\left\{i_{1}, \ldots, i_{k}\right\} \subset\{1, \ldots, N\}$ and evaluate $\left|\operatorname{det} A^{i_{1}, \ldots, i_{k}}\left(x_{1}, \ldots, x_{k}\right)\right|^{2}$. Namely, observe that

$$
\left|\operatorname{det} A^{i_{1}, \ldots, i_{k}}\left(x_{1}, \ldots, x_{k}\right)\right|^{2}=\prod_{p=1}^{k} \frac{1}{\pi \Gamma\left(i_{p}, R^{2}\right)} \mathrm{e}^{-\sum_{p=1}^{k}\left|x_{p}\right|^{2}}\left|V_{i_{1}, \ldots, i_{k}}\left(x_{1}, \ldots, x_{k}\right)\right|^{2},
$$


where $V_{i_{1}, \ldots, i_{k}}\left(x_{1}, \ldots, x_{k}\right)$ is the generalized Vandermonde determinant. Applying the definition of the Schur polynomial (6) yields

$$
V_{i_{1}, \ldots, i_{k}}\left(x_{1}, \ldots, x_{k}\right)=s_{\lambda\left(i_{1}, \ldots, i_{k}\right)}\left(x_{1}, \ldots, x_{k}\right) V_{1, \ldots, k}\left(x_{1}, \ldots, x_{k}\right) .
$$

At this point, we obtain (5) by applying [15, Lemma 3.4], noting that (7) holds.

Next, we wish to determine which $R \geq 0$ to choose in the projection of the point process onto $\mathscr{B}_{R}$. In this regard, we recall that the particle density $\rho_{1}$ of the general Ginibre process is constant, and $\rho_{1}(x)=K(x, x)=1 / \pi$ for $x \in \mathbb{C}$. However, the particle density of the TGPP is not constant. If we denote by $\rho_{n}^{N}$ the $n$th correlation function of the TGPP, then

$$
\rho_{1}^{N}(x)=\frac{1}{\pi} \mathrm{e}^{-|x|^{2}} \sum_{k=0}^{N-1} \frac{|x|^{2 k}}{k !} \quad \text { for } x \in \mathbb{C} .
$$

We note that $\rho_{1}^{N}$ depends only on $|x|$; hence, we write $\rho_{1}^{N}(x)=\rho_{1}^{N}(|x|)$. As can be checked easily, we have $\int_{\mathbb{C}} \rho_{1}^{N}(x) \mathrm{d} \ell(x)=N$ as well as

$$
\rho_{1}^{N}(x) \leq \frac{1}{\pi} \quad \text { for all } x \in \mathbb{C},
$$

and, in fact, it is known that $\rho_{1}^{N}(\sqrt{N} x) \rightarrow(1 / \pi) \mathbf{1}_{\{|x| \leq 1\}}$ as $N \rightarrow \infty$, which is known as the circular law in stochastic matrix theory. Therefore, it appears that it is judicious to project onto $B \sqrt{N}$. To obtain more precise results on the error made by truncating the point process to $\mathscr{B}_{\sqrt{N}}$, we recall the following bounds on $\rho_{1}^{N}$, which were obtained in [4]. For $|x| \leq \sqrt{N}$ and $x=\sqrt{N}-u$,

$$
\rho_{1}^{N}(\sqrt{N}-u) \geq \frac{1}{\pi}-\frac{1}{2 \sqrt{2} u \pi^{3 / 2}} \mathrm{e}^{-2 u^{2}},
$$

as well as for $|z| \geq \sqrt{N}$ and $z=\sqrt{N}+u$,

$$
\rho_{1}^{N}(\sqrt{N}+u) \leq \frac{1}{2 \sqrt{2} u \pi^{3 / 2}} \mathrm{e}^{-2 u^{2}} \text { as } N \rightarrow \infty .
$$

These bounds exhibit the sharp fall of the particle density around $|x|=\sqrt{N}$.

From now on, we consider the truncated Ginibre process of rank $N$ projected onto $B \sqrt{N}$. Note that, by construction, the simulation of the TGPPC is straightforward. Indeed, taking an $N \times N$ matrix with complex Gaussian entries, and conditioning on the points being in $\mathscr{B}_{R}$ yields a DPP with kernel given by (4). Despite the ease of simulation, a significant disadvantage is that the number of points is always less than a fixed parameter $N$. Additionally, we no longer control the number of points as was the case with the TGPP, i.e. there is a random number of points in the compact subset.

At least the latter disadvantage can be lifted by considering the TGPPC conditioned on the number of points being equal to $N$. Since the projection onto $B_{\sqrt{N}}$ of the TGPP takes the form of a DPP of kernel (4), we can easily compute the probability of all the points falling in $B_{\sqrt[N]{N}}$. Indeed, we have

$$
\mathbb{P}_{\mu^{N}}\left(\xi_{\left(B_{\sqrt{N}}\right)^{c}}=\varnothing\right)=\prod_{n=0}^{N-1} \lambda_{n}^{\sqrt{N}}=\prod_{n=0}^{N-1} \frac{\gamma(n+1, N)}{n !}
$$


It can be shown that this probability tends to 0 as $N$ tends to $\infty$. That is, if we are required to simulate the Ginibre process on a compact, conditional on it having $N$ points, the conditioning requires an increasing computation time as $N$ tends to $\infty$.

Instead of simulating the conditioning numerically, we note that the point process resulting from the conditioning is still a DPP with a kernel that may be stated explicitly. Namely, we set

$$
\widetilde{K}^{N}\left(x_{1}, x_{2}\right)=\sum_{n=0}^{N-1} \phi_{n}^{N}\left(x_{1}\right) \overline{\phi_{n}^{N}\left(x_{2}\right)}, \quad x_{1}, x_{2} \in \mathscr{B}_{\sqrt{N}}
$$

and where

$$
\phi_{n}^{N}(x)=\frac{1}{\sqrt{\pi \gamma(n+1, N)}} \mathrm{e}^{-|x|^{2} / 2} x^{n} \mathbf{1}_{\left\{x \in B_{\sqrt{N}}\right\}}, \quad x \in \mathbb{C} .
$$

We emphasize that this is in fact the TGPPC on $\mathscr{B}_{\sqrt{N}}$ conditioned on having $N$ points, this result being due to [6, Theorem 7]. Moreover, the DPP associated with this kernel benefits from the efficient simulation techniques developed in the fundamental algorithm from [6]. Here, the fact that we can explicitly state the projection kernel associated with the conditioning is what ensures the efficiency of the simulation.

Let us now prove proper convergence of the DPP defined by the kernel (8) to the GPP.

Theorem 1. The kernels $\widetilde{K}^{N}$ converge uniformly on compact subsets to $K$ as $N$ tends to $\infty$.

Proof. Take a compact subset $A$ of $\mathbb{C}$, and $\operatorname{set} \operatorname{rad}(A):=\sup \{|z|, z \in A\}$. Then, for $x, y \in A$,

$$
\begin{aligned}
\left|K(x, y)-\widetilde{K}^{N}(x, y)\right| & =\left|\sum_{n=0}^{N-1}\left(\phi_{n}(x) \overline{\phi_{n}(y)}-\phi_{n}^{N}(x) \overline{\phi_{n}^{N}(y)}\right)+\sum_{n=N}^{+\infty} \phi_{n}(x) \overline{\phi_{n}(y)}\right| \\
\leq & \sum_{n=0}^{N-1} \frac{1}{\pi} \operatorname{rad}(A)^{2 n}\left|\frac{1}{\gamma(n+1, N)}-\frac{1}{n !}\right| \mathbf{1}_{\left\{(x, y) \in\left(B_{\sqrt{N}}\right)^{2}\right\}} \\
& +\sum_{n=0}^{N-1} \frac{1}{\pi n !} \operatorname{rad}(A)^{2 n} \mathbf{1}_{\left\{(x, y) \notin\left(\mathcal{B}_{\sqrt{N}}\right)^{2}\right\}}+\sum_{n=N}^{\infty} \frac{1}{\pi n !} \operatorname{rad}(A)^{2 n} .
\end{aligned}
$$

The third term in this inequality tends to 0 as the remainder of a convergent series, and the second term also tends to 0 by dominated convergence. Concerning the first term, we need more precise arguments, so let us start by writing it as

$$
\begin{aligned}
& \sum_{n=0}^{\infty} \frac{1}{\pi} \operatorname{rad}(A)^{2 n} \frac{1}{\gamma(n+1, N)} \mathbf{1}_{\left\{\left(z_{1}, z_{2}\right) \in\left(\mathscr{B}_{\sqrt{N}}\right)^{2}\right\}} \mathbf{1}_{\{n \leq N-1\}} \\
& -\sum_{n=0}^{N-1} \frac{1}{\pi n !} \operatorname{rad}(A)^{2 n} \mathbf{1}_{\left\{\left(z_{1}, z_{2}\right) \in\left(\mathbb{B}_{\sqrt{N}}\right)^{2}\right\}},
\end{aligned}
$$

and noting that $\gamma(n+1, N) \rightarrow n$ ! as $N$ tends to $\infty$. Therefore, in order to conclude, we wish to exhibit a summable bound to the summand of the first term in (9). To this end, we write

$$
\frac{1}{\gamma(n+1, N)} \mathbf{1}_{\{n \leq N-1\}} \leq \frac{1}{\gamma(n+1, n+1)},
$$


and note that

$$
\frac{1}{\gamma(n+1, n+1)}=\frac{1}{n ! \mathbb{P}\left(\sum_{k=1}^{n+1} X_{k} \leq n+1\right)} \sim \frac{2}{n !} \quad \text { as } n \rightarrow \infty,
$$

where we define $f_{n} \sim g_{n}$ as $n \rightarrow \infty$ to mean that $f_{n} / g_{n} \rightarrow 1$ as $n \rightarrow+\infty$, and where $X_{1}, \ldots, X_{n}$ are independent exponential random variables of parameter 1 defined on a probability space $(\Omega, \mathcal{A}, \mathbb{P})$. In (10), we have used the fact that $\gamma(a, R) / \Gamma(a)$ is the cumulative distribution function of a $\Gamma(a, R)$ random variable, $a>0$, and $R \geq 0$. The asymptotic behavior in (11) follows from the application of the central limit theorem to $X_{1}, \ldots, X_{n}$. Hence,

$$
\begin{aligned}
\sum_{n=0}^{\infty} \frac{1}{\pi} \operatorname{rad}(A)^{2 n} \frac{1}{\gamma(n+1, N)} \mathbf{1}_{\left\{\left(z_{1}, z_{2}\right) \in\left(\mathcal{B}_{\sqrt{N}}\right)^{2}\right\}} \mathbf{1}_{\{n \leq N-1\}} & \leq \sum_{n=0}^{\infty} \frac{1}{\pi \gamma(n+1, n+1)} \operatorname{rad}(A)^{2 n} \\
& <\infty
\end{aligned}
$$

which means that by Lebesgue's dominated convergence theorem, (9) tends to 0 as $N$ tends to $\infty$. Therefore, $\sup _{x, y \in A}\left|K(x, y)-\widetilde{K}_{N}(x, y)\right| \rightarrow 0$ as $N \rightarrow \infty$.

As a consequence of Theorem 1 and in [15, Proposition 3.10], the DPP associated with $\widetilde{K}^{N}$ converges weakly to the GPP.

We now return to the problem of simulating the DPP with the kernel given by (8). As it is a projection DPP, it is efficiently simulated according to the algorithm described in detail in [6]. However, the first step of generating the Bernoulli random variables is not necessary, as we are working conditional on there being $N$ points. Finally, we note that the method described in this section yields a DPP on $B_{\sqrt{N}}$. In order to simulate on $\mathscr{B}_{R}$, we need to apply a homothetic transformation to the $N$ points, which translates to a homothety on the eigenvectors. To summarize and in order to make this paper more self-contained, the simulation algorithm of the TGPPC of the kernel (8) on a centered ball of radius $R \geq 0$ is as follows.

Algorithm 1. (Simulation of the TGPPC.) The algorithm is as follows.

Define $\phi_{k}(x)=\left(N / \pi R^{2} \gamma(k+1, N)\right) \mathrm{e}^{-\left(N / 2 R^{2}\right)|x|^{2}}\left(N x / R^{2}\right)^{k}$ for $x \in B_{R}$ and $0 \leq k \leq N-1$.

Define $v(x):=\left(\phi_{0}^{N}(x), \ldots, \phi_{N-1}^{N}(x)\right)$ for $x \in B_{R}$.

Sample $X_{N}$ from the distribution with density $p_{N}(x)=\|v(x)\|^{2} / N, x \in \mathscr{B}_{R}$.

Set $e_{1}=v\left(X_{N}\right) /\left\|v\left(X_{N}\right)\right\|$.

for $i=N-1 \rightarrow 1$ do

Sample $X_{i}$ from the distribution with density

$$
p_{i}(x)=\frac{1}{i}\left[\|\boldsymbol{v}(x)\|^{2}-\sum_{j=1}^{N-i}\left|\boldsymbol{e}_{j}^{*} v(x)\right|^{2}\right]
$$

Set $w_{i}=v\left(X_{i}\right)-\sum_{j=1}^{N-i}\left(e_{j}^{*} v\left(X_{i}\right)\right) e_{j}$, and $e_{N-i+1}=w_{i} /\left\|w_{i}\right\|$.

end for

return $\left(X_{1}, \ldots, X_{N}\right)$. 

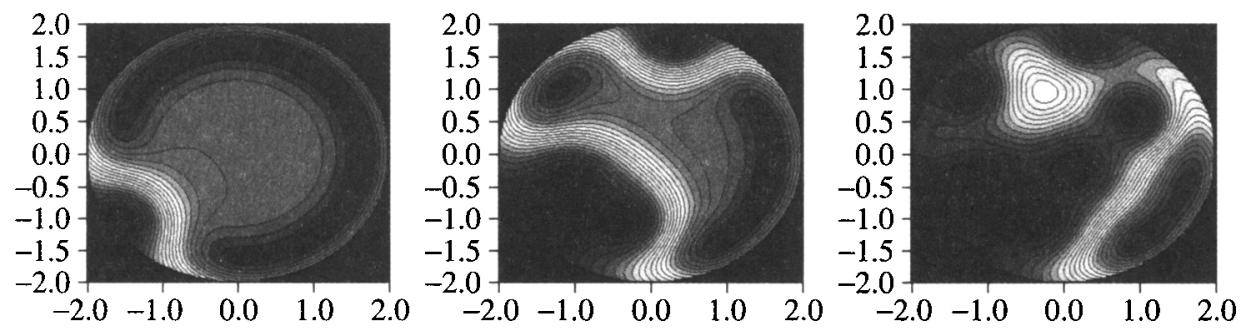

FIGURE 1: Probability density in the simulation of the TGPPC.

The resulting process is a DPP with kernel (8). Its support is on the compact set $\mathscr{B}_{R}$ and has $N$ points almost surely. We now give a brief example of the results of Algorithm 1 applied for $R=2$ and $N=9$ at steps $i=8, i=5$, and $i=2$, respectively. We plot the densities used for the simulation of the next point in Figure 1. We note here that the density is supported on $\mathscr{B}_{R}$.

This DPP presents the advantage of being easy to use in simulations, as well as having $N$ points almost surely. Moreover, Theorem 1 proves its convergence to the GPP as $N$ tends to $\infty$.

We end this section by mentioning the difficulties arising in the simulation under the density $p_{i}, 1 \leq i \leq N-1$. As is remarked in [8], in the general case, we have no choice but to simulate by rejection sampling and the GPP is no different (except in the step $i=N-1$, where $p_{i}$ is the density of a Gaussian random variable). Therefore in practice, we draw a uniform random variable $u$ on $\mathscr{B}_{R}$ and choose $p_{i}(u) / \sup _{y \in B_{R}} p_{i}(y)$. Note that the authors in [8] give a closed form bound on $p_{i}$, which is given by

$$
p_{i}(x) \leq \frac{1}{i} \min _{i+1 \leq k \leq N}\left(\widetilde{K}^{N}(x, x)-\frac{\left|\widetilde{K}^{N}\left(x, X_{k}\right)\right|^{2}}{\widetilde{K}^{N}\left(X_{k}, X_{k}\right)}\right), \quad x \in \mathbb{C},
$$

where $X_{i+1}, \ldots, X_{N}$ is the result of the simulation procedure up to step $i$. In practice however, the error made in the previous inequality is not worth the gain made by not evaluating $\sup _{y \in \mathscr{B}_{R}} p_{i}(y)$. Therefore, we have chosen not to use (12).

Additionally, the simulation techniques in [8] use the Fourier basis. In this case, it is clear that $p_{i} \leq 1$, and this bound is quite efficient. In the case of the GPP, implementing a mathematical bound slows down the simulation procedure as it is in our experience often far from $\sup _{y \in B_{R}} p_{i}(y)$.

\section{Acknowledgement}

The authors wish to thank the anonymous referee for the insightful comments leading to the present version of the paper.

\section{References}

[1] Anderson, G. W., Guionnet, A. And Zeitouni, O. (2010). An Introduction to Random Matrices (Camb. Stud. Adv. Math. 118). Cambridge University Press.

[2] Bornemann, F. (2011). On the scaling limits of determinantal point processes with kernels induced by SturmLiouville operators. Preprint. Available at http://arxiv.org/abs/1104.0153.

[3] Decreusefond, L., Flint, I. And Vergne, A. (2013). Efficient simulation of the Ginibre point process. Preprint. Available at http://arxiv.org/abs1310.0800.

[4] Ginibre, J. (1965). Statistical ensembles of complex, quaternion, and real matrices. J. Math. Phys. 6, 440-449. 
[5] Heineman, E. R. (1929). Generalized Vandermonde determinants. Trans. Amer. Math. Soc. 31, $464-476$.

[6] Hough, J. B., Krishnapur, M., Peres, Y. ANd Virág, B. (2006). Determinantal processes and independence. Prob. Surv. 3, 206-229.

[7] Hough, J. B., Krishnapur, M., Peres, Y. AND VIRÁG, B. (2009). Zeros of Gaussian Analytic Functions and Determinantal Point Processes (Univ. Lecture Ser. 51). American Mathematical Society, Providence, RI.

[8] LavanCier, F., Møller, J. ANd RubaK, E. (2015). Determinantal point process models and statistical inference. J. R. Statist. Soc. B 77, 853-877.

[9] Le CaËr, G. AND Delannay, R. (1993). The administrative divisions of mainland France as 2D random cellular structures. J. Phys. I 3, 1777-1800.

[10] LE CAËR, G. AND Ho, J. S. (1990). The Voronoi tessellation generated from eigenvalues of complex random matrices. J. Phys. A 23, 3279.

[11] MaCCHI, O. (1975). The coincidence approach to stochastic point processes. Adv. Appl. Prob. 7, 83-122.

[12] Miyoshi, N. AND Shirai, T. (2014). A cellular network model with Ginibre configurated base stations. Adv. Appl. Prob. 46, 832-845.

[13] Scardicchio, A., Zachary, C. E. and Torquato, S. (2009). Statistical properties of determinantal point processes in high-dimensional Euclidean spaces. Phys. Rev. E (3) 79, 041108.

[14] ShiraI, T. (2006). Large deviations for the fermion point process associated with the exponential kernel. $J$. Statist. Phys. 123, 615-629.

[15] Shirai, T. and Takahashi, Y. (2003). Random point fields associated with certain Fredholm determinants. I. Fermion, Poisson and boson point processes. J. Funct. Anal. 205, 414 463.

[16] Soshnikov, A. (2000). Determinantal random point fields. Uspekhi Mat. Nauk 55, 107-160.

[17] Tamura, H. AND Ito, K. R. (2006). A canonical ensemble approach to the fermion/boson random point processes and its applications. Commun. Math. Phys. 263, 353-380.

[18] ToRrisi, G. L. AND LEONARDI, E. (2014). Large deviations of the interference in the Ginibre network model. Stoch. Syst. 4, 173-205.

[19] Vergne, A., Flint, I., Decreusefond, L. And Martins, P. (2014). Disaster recovery in wireless networks: a homology-based algorithm. In ICT 2014 (Lisbon), pp. 226-230. 